\title{
Web Search of Fashion Items with Multimodal Querying
}

\author{
Katrien Laenen \\ KU Leuven \\ Human Computer Interaction \\ Heverlee, Belgium \\ katrien.laenen@kuleuven.be
}

\author{
Susana Zoghbi \\ KU Leuven \\ Human Computer Interaction \\ Heverlee, Belgium \\ susana.zoghbi@kuleuven.be
}

\author{
Marie-Francine Moens \\ KU Leuven \\ Human Computer Interaction \\ Heverlee, Belgium \\ sien.moens@kuleuven.be
}

\begin{abstract}
In this paper, we introduce a novel multimodal fashion search paradigm where e-commerce data is searched with a multimodal query composed of both an image and text. In this setting, the query image shows a fashion product that the user likes and the query text allows to change certain product attributes to fit the product to the user's desire. Multimodal search gives users the means to clearly express what they are looking for. This is in contrast to current e-commerce search mechanisms, which are cumbersome and often fail to grasp the customer's needs. Multimodal search requires intermodal representations of visual and textual fashion attributes which can be mixed and matched to form the user's desired product, and which have a mechanism to indicate when a visual and textual fashion attribute represent the same concept. With a neural network, we induce a common, multimodal space for visual and textual fashion attributes where their inner product measures their semantic similarity. We build a multimodal retrieval model which operates on the obtained intermodal representations and which ranks images based on their relevance to a multimodal query. We demonstrate that our model is able to retrieve images that both exhibit the necessary query image attributes and satisfy the query texts. Moreover, we show that our model substantially outperforms two state-of-the-art retrieval models adapted to multimodal fashion search.
\end{abstract}

\section{ACM Reference format:}

Katrien Laenen, Susana Zoghbi, and Marie-Francine Moens. 2018. Web Search of Fashion Items with Multimodal Querying. In Proceedings of WSDM 2018: The Eleventh ACM International Conference on Web Search and Data Mining, Marina Del Rey, CA, USA, February 5-9, 2018 (WSDM 2018), 9 pages. https://doi.org/10.1145/3159652.3159716

\section{INTRODUCTION}

Today's consumers have become very exigent. When shopping online, they have in mind a specific clothing item in a particular color and style, and they want to find it without too much effort. However, current e-commerce search mechanisms are often too limited to provide this kind of service. A common way for searching

\footnotetext{
${ }^{1}$ Image material adapted from www.amazon.com

Permission to make digital or hard copies of all or part of this work for personal or classroom use is granted without fee provided that copies are not made or distributed for profit or commercial advantage and that copies bear this notice and the full citation on the first page. Copyrights for components of this work owned by others than ACM must be honored. Abstracting with credit is permitted. To copy otherwise, or republish, to post on servers or to redistribute to lists, requires prior specific permission and/or a fee. Request permissions from permissions@acm.org.

WSDM 2018, February 5-9, 2018, Marina Del Rey, CA, USA

(c) 2018 Association for Computing Machinery.

ACM ISBN 978-1-4503-5581-0/18/02 .\$15.00

https://doi.org/10.1145/3159652.3159716
}



Find similar images but short and with lace

Figure 1: Example of a multimodal query ${ }^{1}$. It consists of a query image and a query text that alters the query image. The query text mentions two fashion attributes: short and lace. The query image is knee length and does not have a lace type appearance.

products in a webshop is to navigate through a product category hierarchy. Users end up in a subcategory which they need to search completely, which is time-consuming. They need to go through many irrelevant products, without the guarantee of actually finding the product they are looking for. To narrow down the search, users can sometimes select certain filters. However, desired product attributes might not be amongst the available filters. With a text-based search approach, users can describe the desired product by entering keywords into a search bar. The webshop then finds relevant products by matching these keywords to the words in the product descriptions. It is often difficult for users to write the right keywords that will induce the search engine to provide the products they are interested in. For example, some users might be interested in "jeans with holes", but the relevant products are described as "distressed jeans". Additionally, this approach hampers the search for product attributes which are not mentioned in the product descriptions. Alternatively but rarely, webshops offer image-based search where the user uploads an image of the desired product and receives visually similar products. Recently, there is an increasing interest of users in this kind of image-based search. One of the main reasons is the growing usage of visual social media such as Pinterest and Instagram, where users see products they want to buy. Using an image as a query allows users to convey much more information about the desired product than with a textual query. Additionally, another advantage of image-based search over text-based search is that the language of images is universal. However, the user might be interested in changing or adding attributes to the product in the query image to obtain very specific results. For example, for an image of a red dress, the user likes the sleeve length to be different. 
Currently, it is not possible to search for products using both an image and text, where the text is used to narrow down the search with fine-grained attributes.

We propose multimodal search where a query is composed of both an image and a text that modifies the image or adds attributes to it (Figure 1). Relevant images are those which are similar to the query image, but with the attributes that the query text changed or added. Since multimodal search allows users to search with images and uses text as a mechanism to easily manipulate image properties, it is likely to become a very popular search method. To answer a multimodal query, it should be possible to identify the fashion attributes in the query image and text to ensure the retrieval of images in which these attributes are exhibited. Hence, multimodal search requires fine-grained segmentation methods for fashion images and product descriptions, and a mechanism to determine when a visual and textual fashion attribute represent the same concept. For example, for the multimodal query in Figure 1 , we need to identify the attributes of the query image, i.e., the kind of neckline, sleeve length, style, etc., and a way to know if a candidate image also displays the attributes requested by the query text, i.e., if it is short and contains lace.

In this paper, we will consider the product category of dresses. We segment fashion images and texts into image regions and phrases showing respectively visual and textual fashion attributes. We learn intermodal representations for these visual and textual attributes which capture their semantic similarity. With a neural network, we infer a common, multimodal space where a fashion image region and phrase have a large inner product if they represent the same fashion attribute. Then, we develop a multimodal retrieval model which operates on the obtained intermodal representations. Note that although this work focuses on the fashion domain, the proposed techniques are valuable for processing other product data as well (e.g., furniture, toys or food) where data consists of pairs of images and texts, with texts that describe product properties which are either shown in the corresponding image or can be inferred from it.

The contributions of our work are:

- We introduce a new search paradigm for e-commerce product retrieval constituting an improved shopping experience.

- Our model provides a mechanism to reason with both images and language, i.e., we can query a target collection using a combination of an image and text as query. The text can be used to modify specific attributes in the image, providing more fine-grained and relevant results.

- We largely improve the results of two state-of-the-art retrieval models adapted to multimodal search.

- We provide an analysis of the neural network based alignment model trained in Laenen et al. [12] to align fashion attributes in images and their textual descriptions.

The remainder of this paper is structured as follows. In Section 2 we review existing work related to the subject. Section 3 provides a detailed description of our model architecture. Section 4 describes the experiments conducted in this work. Next, the results of these experiments are presented in Section 5. Finally, section 6 gives the final conclusions and directions for future work.

\section{RELATED WORK}

Image search aims to retrieve relevant images to a textual or visual query. In [18], Zoghbi et al. experiment with canonical correlation analysis (CCA) and bilingual latent Dirichlet allocation (BiLDA) to realize cross-modal fashion search from a textual query to images and from an image query to text. In [12], cross-modal search of fashion items is achieved with intermodal representations for visual and textual fashion attributes inferred with a neural network alignment model. It is a short workshop paper which discusses initial experiments by the authors of this paper and which explains the objective functions used to infer the latent alignment of the visual and textual fashion attributes. Meanwhile, this work explores the capabilities of the inferred intermodal representations in a multimodal search instead of a cross-modal search setting, proposes a retrieval model for multimodal search, and reveals more details about the neural network alignment model. Opposed to textual queries, visual queries capture rich information about desired product properties. Recently, with the rise of deep convolutional neural networks, image search with visual queries or content-based image retrieval (CBIR) has attracted a lot of interest. Several systems have been developed that, given an image as a query, return products in webshops which are similar or identical to the product in the provided image $[1,7,9,11,16]$. In contrast to our work, these systems do not allow users to add text to change certain product properties.

Multimodal search with queries composed of an image and a text is a novel search paradigm which up until now has received little attention in the literature. In [17], an attribute manipulation network is developed which learns to construct the visual representation of the desired fashion item by properly manipulating the visual representation of the multimodal query image with the textual attributes in the multimodal query text, and retrieves images with representations similar to the constructed visual representation. In contrast, we use a neural network to infer the semantic relationship between visual and textual attributes in a joint embedding space, and propose a multimodal retrieval model which ranks images based on their semantic similarity to the query text and their visual similarity to the query image. In [6], fashion images and their corresponding product descriptions are jointly modeled in a multimodal embedding space. Then, spatial-semantic representations are derived for all fashion attributes and used to cluster the attributes (e.g., V-neck) into attribute types (e.g., neckline). Finally, the multimodal embedding space is decomposed into multiple subspaces, one for each attribute type. These subspaces are used to facilitate so-called attribute-feedback product retrieval, where a multimodal query consists of an image and a text denoting a single attribute to either add to or change in the query image, and the retrieval model is based on adding and subtracting spatial-semantic representations of attributes. In contrast, we infer only one multimodal embedding space which uncovers the latent alignment on the attribute level, where the resulting intermodal representations are used in a multimodal search paradigm that allows multimodal query texts which simultaneously change and add multiple query image attributes. In [4], multimodal queries are introduced in category-swap imagebased retrieval using only three general object categories that can modify the query and exploring a retrieval model that is inspired by [13]. In [13], semantic inference is performed by adding and 
subtracting word embeddings. In this work, we will do the same with intermodal representations of fashion attributes in one of our multimodal search baselines.

Beyond product search, multimodal approaches have also become more popular due to the abundance of multimodal data and the recent advances in deep learning to process visual and language data. More precisely, several systems have been developed for image-text alignment where image descriptions are generated with a recurrent neural network (RNN) [3, 10, 14, 15]. In this work, our interest lies not in the generation of image annotations, but in the alignment of attributes in visual and language data, which is essential for the multimodal retrieval model.

\section{METHODOLOGY}

In multimodal search, a query consists of an image of a product together with text to alter or add certain product attributes. The fashion domain presents large variations of fine-grained fashion attributes: colors, shapes, patterns, textures, etc. We learn intermodal representations for these fashion attributes in images and texts, which reflect their semantic similarity.

First, we describe how we segment fashion images and product descriptions into fragments showing respectively the visual and textual fashion attributes. Next, we discuss the neural network and objective function used to obtain intermodal representations for fashion attributes. Finally, we elaborate on how multimodal search is achieved with the acquired intermodal representations.

\subsection{Visual and Textual Attribute Detection}

E-commerce product descriptions tend to be noisy and incomplete. To acquire the textual fashion attributes, we follow the approach of Zoghbi et al. [18] and filter the product descriptions with the glossary of the online clothing shop Zappos ${ }^{2}$. This process only retains fashion-related phrases and removes most noise. However, remaining phrases can still describe properties of the dress which are not displayed, e.g., all possible colors. After filtering, the remaining Zappos phrases are considered as the textual fashion attributes and form the text fragments. The text fragments are represented with word embeddings [13] trained on the Amazon Dresses dataset.

E-commerce images display clothing items straight, clearly and completely on white backgrounds, sometimes worn by a model. This tells us the approximate location of each part of a clothing item and thus of each visual fashion attribute. We implement the dress segmentation method of Laenen et al. [12] which exploits this fact to generate 7 image fragments corresponding to dress regions where visual fashion attributes are likely to be found. These 7 image fragments show respectively the full dress, the top, the full skirt, the part of the skirt above the knee, the neckline, the left sleeve and the right sleeve. The image fragments are represented with the BVLC CaffeNet CNN Model [8]. Their representations are acquired as the activation weights of the last fully connected layer before the softmax layer, which have dimension 4096 in the CNN architecture.

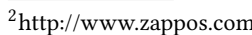

\subsection{Common Embedding Space for Visual and Textual Attributes}

With the current image and text fragment representations, we have no way of knowing when they correspond to the same fashion attribute. Therefore, we use a neural network to induce a common, multimodal space where the inner product of an image fragment and text fragment measures their semantic similarity. A positive inner product indicates that they represent the same fashion attribute. When their inner product is negative, they are considered different. The neural network learns parameters $\theta=\left\{W_{v}, b_{v}, W_{s}, b_{s}\right\}$ to project an image fragment $\hat{v}_{i}$ and text fragment $\hat{s}_{j}$ to their intermodal representations $v_{i}$ and $s_{j}$ in the common, multimodal space:

$$
\begin{aligned}
& v_{i}=W_{v} \hat{v}_{i}+b_{v} \\
& s_{j}=f\left(W_{s} \hat{s}_{j}+b_{s}\right) .
\end{aligned}
$$

Activation function $f$ is set to the rectified linear unit (ReLU) which computes $f(x)=\max (0, x)$. $W_{v}$ has dimensions $h \times 4096$ and $W_{s}$ has dimensions $h \times \operatorname{dim}$, where $h$ is the size of the common, multimodal space and dim is the dimension of the word embeddings. Parameters $b_{v}$ and $b_{s}$ are bias terms.

We train the neural network with objective function $C(\theta)$ composed of the fragment alignment objective $C_{F}(\theta)$ [10], global ranking objective $C_{G}(\theta)$ [10] and image cluster consistency objective $C_{I}(\theta)[12]:$

$$
C(\theta)=C_{F}(\theta)+\gamma C_{I}(\theta)+\beta C_{G}(\theta)+\alpha\|\theta\|_{2}^{2},
$$

where $\theta$ refers to the network parameters and $\alpha, \beta$ and $\gamma$ are hyper parameters cross-validated on a validation set.

The fragment alignment objective $C_{F}(\theta)$ [10] uses fragment cooccurrence information to infer the semantic similarity $v_{i}^{T} s_{j}$ of image fragment $v_{i}$ and text fragment $s_{j}$. It is formulated as:

$$
\begin{aligned}
& C_{F}(\theta)=\min _{y_{i j}} C_{0}(\theta) \\
& C_{0}(\theta)=\sum_{i} \sum_{j} \max \left(0,1-y_{i j} v_{i}^{T} s_{j}\right) \\
& \text { subject to } \sum_{i \in p_{j}} \frac{y_{i j}+1}{2} \geq 1 \forall j \\
& y_{i j}=-1 \forall i, j \text { subject to } m_{v}(i) \neq m_{s}(j) \\
& \text { and } y_{i j} \in\{-1,1\} .
\end{aligned}
$$

Here, variable $y_{i j}$ reflects whether $v_{i}$ and $s_{j}$ correspond to the same fashion attribute $\left(y_{i j}=1\right)$ or not $\left(y_{i j}=-1\right), p_{j}$ is the bag of image fragments occurring with $s_{j}$, and $m_{v}(i)$ and $m_{s}(j)$ return the index of the image-text pair that respectively $v_{i}$ and $s_{j}$ belong to.

The global ranking objective $C_{G}(\theta)$ [10] derives semantic similarity from global image-text correspondence: 


$$
\begin{aligned}
C_{G}(\theta)=\sum_{k}[\underbrace{\sum_{l} \max \left(0, S_{k l}-S_{k k}+\Delta\right)}_{\text {rank images }} \\
+\underbrace{\sum_{l} \max \left(0, S_{l k}-S_{k k}+\Delta\right)}_{\text {rank texts }} .
\end{aligned}
$$

It encourages corresponding image-text pairs $(k=l)$ to have a higher total semantic similarity score than non-corresponding pairs $(k \neq l)$, by a margin $\Delta$. The total semantic similarity score $S_{k l}$ of an image $k$ and text $l$ is computed based on the semantic similarity scores of their respective fragments $f_{k}$ and $f_{l}$ :

$$
S_{k l}=\frac{1}{\left(\left|f_{l}\right|+n\right)} \sum_{j \in f_{l}} \max _{i \in f_{k}} v_{i}^{T} s_{j},
$$

with $n$ a smoothing term.

The image cluster consistency objective $C_{I}(\theta)$ [12] uses image fragment similarity information to recover semantic similarity information lost due to noise and incompleteness of product descriptions. It is given by:

$$
C_{I}(\theta)=\sum_{m=1}^{M} \sum_{i} \sum_{j}\left(1-\frac{\hat{v}_{i}^{T} \hat{c}_{i}}{\left\|\hat{v}_{i}\right\|\left\|\hat{c}_{i}\right\|}\right)\left|v_{i}^{T} s_{j}-c_{i}^{T} s_{j}\right| .
$$

where $M$ is the number of image-text pairs in the training set, $\hat{v}_{i}$ is a training image fragment, and $\hat{c_{i}}$ is an image fragment which is visually similar to $\hat{v}_{i}$.

\subsection{Multimodal Search}

In multimodal search, a query is composed of both an image and a text, where the query text is used to modify parts of the query image. The query text can request to change certain fashion attributes (e.g., ask for long sleeves when the dress in the query image is sleeveless) and/or to add fashion attributes to the image (e.g., ask to add rhinestones). For example, Figure 1 shows a multimodal query that both requests to change the dress length (from knee length to short) and to add ornaments (lace) to the dress. We do not support multimodal queries where the query text removes fashion attributes from the query image, as this is less in accordance with how users search.

We propose a multimodal retrieval model which ranks images $i_{c}$ based on their relevance to a multimodal query $q$, consisting of query image $i_{q}$ and query text $t_{q}$. Query image $i_{q}$ and candidate image $i_{c}$ are segmented with the image segmentation technique described above, producing image fragments $\hat{v}_{i, q}$ and $\hat{v}_{i, c}$ for respectively the query image and candidate image $(i=1, \ldots, 7)$. Query text $t_{q}$ is filtered with the Zappos glossary to acquire one or more text fragments $\hat{s}_{j, q}$ referring to fashion attributes to be either added or interchanged with current attributes of the query image. Image and text fragments are represented with the intermodal representations $v_{i, q}, v_{i, c}$ and $s_{j, q}$ as inferred with the neural network. We use cosine similarity, i.e., the inner product scaled by magnitude, to measure both the visual similarity of two image fragments and the semantic similarity of an image and text fragment. The cosine similarity takes the sign of the inner product. Consequently, the cosine similarity of an image and text fragment will be positive if they represent the same fashion attribute, and will be negative otherwise. To find relevant images for a multimodal query $\left\{i_{q}, t_{q}\right\}$, we compute the similarity of $\left\{i_{q}, t_{q}\right\}$ with all candidate images $i_{c}$ as follows:

$$
\begin{aligned}
\operatorname{Sim}\left(i_{q}, t_{q}, i_{c}\right) & =\operatorname{Sim}\left(i_{q}, i_{c}\right)+\operatorname{Sim}\left(t_{q}, i_{c}\right) \\
\operatorname{Sim}\left(i_{q}, i_{c}\right) & =\sum_{i} \frac{v_{i, q}^{T} v_{i, c}}{\left\|v_{i, q}\right\|\left\|v_{i, c}\right\|} \\
\operatorname{Sim}\left(t_{q}, i_{c}\right) & =\left\{\begin{array}{l}
\sum_{j} \sum_{i} \max \left(0, w \frac{v_{i, c}^{T} s_{j, q}}{\left\|v_{i, c}\right\|\left\|s_{j, q}\right\|}\right), \\
\text { if } \sum_{i} \max \left(0, w \frac{v_{i, c}^{T} s_{j, q}}{\left\|v_{i, c}\right\|\left\|s_{j, q}\right\|}\right)>0 \forall j \\
-\infty, \\
\text { otherwise. }
\end{array}\right.
\end{aligned}
$$

We consider an image $i_{c}$ relevant if it resembles query image $i_{q}$ and satisfies query text $t_{q}$ (Eq. 12). Candidate image $i_{c}$ resembles query image $i_{q}$ if image fragments $v_{i, c}$ and $v_{i, q}$ of corresponding image parts $i$ are visually similar (Eq. 13). Candidate image $i_{c}$ satisfies query text $t_{q}$ if every query text fragment $s_{j, q}$ is shown in at least one image fragment $v_{i, c}$ of the candidate image (i.e., if every $s_{j, q}$ has a positive cosine similarity with at least one $v_{i, c}$ ). Consequently, candidate images which are similar to the query image but which do not have (some of) the attributes requested in the query text will get a similarity of $-\infty$. The more candidate image fragments display query text fragments, the more relevant we consider the candidate image to be (Eq. 14). In Eq. 14, weighting term $w$ regulates how strongly we want the query text to modify the query image. A smaller weight will assign a higher relevance to candidate images which are similar to the query image, while a larger weight will favor images which satisfy the query text (but which might be less similar to the query image). Hence, we should find a weight $w$ that provides a nice balance between both.

\subsection{Baseline Retrieval Models for Multimodal Search}

We compare the proposed multimodal retrieval model to three baseline retrieval models for multimodal search. As a first baseline, we simply retrieve images with a high cosine similarity with the intermodal representation of the first image fragment of the query image (i.e., the image fragment showing the full dress) and ignore the query text. The second baseline is the standard multimodal retrieval model [13], which creates an intermodal representation of the desired image based on the multimodal query, and retrieves candidate images which are visually similar to the desired image. The intermodal representation of the desired image is equal to the intermodal representation of the first image fragment of the query image, plus the intermodal representations of the query text fragments, minus the intermodal representations of the attributes that the query text fragments will replace. For example, for the query in Figure 1, the standard multimodal retrieval model takes the intermodal representation of the first image fragment of the query image, adds the intermodal representations of "short" and 
"lace", and subtracts the intermodal representation for "knee length". Then, it retrieves candidate images with a high cosine similarity with the resulting vector. Our third baseline is the image-to-textto-image multimodal retrieval model. The image-to-text-to-image retrieval model starts by generating a textual description $l$ of the multimodal query. It does so by first listing for each query image fragment the 3 Zappos phrases with the highest semantic similarity score, then removing from this list the Zappos phrases referring to attributes to be replaced by the query text, and finally appending the query text fragments. Afterwards, the generated textual description $l$ is used to search satisfying images by retrieving images $k$ with the highest total semantic similarity $S_{k l}$ with textual description $l$ (Eq. 10). Note that the second and third baseline retrieval models require the user to also specify in the query text which fashion attribute(s) to replace. For example, for the multimodal query in Figure 1, the query text becomes "Find similar images but short instead of knee length and with lace”.

\section{EXPERIMENTAL SETUP}

\subsection{Dataset}

We train our model on the Amazon Dresses dataset, which we obtained from Zoghbi et al. [18]. This dataset consists of 53689 image-text pairs, where each pair consists of an image of a dress and the corresponding product description. The images show dresses for different occasions, e.g., bridesmaid, casual, wear to work, etc. Hence, they display a large variety of fine-grained fashion attributes. Their corresponding texts roughly describe the image content and can be noisy.

We use 48689 image-text pairs for training, 4000 for validation and 1000 for testing in the same setup as in [18]. During testing, we evaluate the quality of the inferred intermodal representations in a multimodal retrieval setting. We collected 100 multimodal queries from 10 test subjects. Two men and eight women each created 10 multimodal queries reflecting real search requests encountered while browsing the dataset. The query images are images from the test set. The query texts consist of Zappos phrases denoting fashion attributes to either add to or change in the query image. In our dataset, the number of Zappos phrases in a query text ranges from one to three, but in general a query text can contain any number of Zappos phrases. The Zappos glossary consists of more than 200 phrases, 47 of which are used in the multimodal queries. As the ground truth for a multimodal query, we consider all test set images in which all query text attributes are either added or interchanged with the necessary query image attributes and all other query image attributes are preserved. However, since the test dataset only consists of 1000 images displaying a large variety of visual attributes, we can expect that for some multimodal queries there are no relevant images.

\subsection{Experiments}

On the image side, we detect image fragments with the image segmentation technique described above. To find visually similar image fragments, we cluster the image fragments in $C$ clusters with $\mathrm{k}$-means clustering based on cosine distance. As in [12], we take $C=10000$. On the text side, we filter the product descriptions with the Zappos glossary. The remaining phrases function as text fragments. We represent the text fragments with 300-dimensional word embeddings which we train with the Skip-gram model on the Amazon Dresses dataset. Afterwards, the neural network induces a $h$-dimensional common, multimodal space, where image and text fragments have intermodal representations that capture their semantics. As in [12], we choose a common embedding space dimension $h$ of 1000, a smoothing term $n$ in $S_{k l}$ of 10, a margin $\Delta$ in $C_{G}(\theta)$ of 40 , and factors $\beta$ and $\gamma$ in $C(\theta)$ of 0.50 and 0.25 respectively. The neural network is trained with the proposed objective function, which we optimize with stochastic gradient descent with mini-batches of 100 , a fixed learning rate of $10^{-5}$, and a momentum of 0.90 during 20 epochs. Finally, we use the inferred intermodal representations in the proposed multimodal retrieval model. Here, a regulator term $w$ of 2.5 was found to work best based on a small validation set. We investigate both the effect of including and omitting the image cluster consistency objective $C_{I}(\theta)$ when learning the intermodal representations on our multimodal search results. When $C_{I}(\theta)$ is included in the objective function, we consider both the medoid and centroid of the cluster of $\hat{v}_{i}$ as suitable image fragments to fulfill the role of $\hat{c_{i}}$.

\subsection{Evaluation}

In multimodal search, we retrieve for each multimodal query the top $K$ most likely test set images. We evaluate by computing precision@K for $K=1,5$ and mean average precision (MAP). As the ground truth for a multimodal query, we consider all images in which all attributes requested in the query text are either added or interchanged with the necessary query image attributes and all other attributes of the query image are still present. For example, for the multimodal query in Figure 1, a relevant image has the following fashion attributes: "white", "scoop", "sleeveless", "A-line", "casual" (attributes of query image), "short" and "lace" (attributes of query text). This kind of evaluation is rather subjective and strict. It is subjective because sometimes it is unclear if a required fashion attribute is present or not. For example, the color of the retrieved image might be very similar, but not identical, to the one in the query image. Also, there are some multimodal queries which request a certain dress, but more suitable for another occasion (e.g., wear to work). The relevance of the retrieved images for such queries can be judged differently depending on the assessor. When a certain fabric is requested, the product descriptions of the retrieved images are consulted to determine the presence of the fabric, as this is often unclear from the image material. We measured annotator agreement on precision@1 with Cohen's $\kappa$ which yielded a $\kappa=0.45$. This can be considered as a fair agreement. The evaluation is also rather strict. It will consider a retrieved image irrelevant even if it has all but one of the required fashion attributes. Furthermore, we compute precision@K for $K=5$ for each of the 47 query text attributes individually, and macro-average the obtained precision@K yielding the macro $A P @ K$ for $K=5$ across all 47 query text attributes. We compare the results of our proposed multimodal retrieval model to the three baseline retrieval models.

Since our quantitative evaluation is rather subjective and strict, we also provide a qualitative evaluation of our results. We present examples of multimodal queries and calculate precision@K for 
Table 1: Multimodal search results. Comparison of performance of different retrieval models. $P @ K$ is precision@K and macro $A P @ K$ is the average attribute precision@ $K$ over all textual attributes.

\begin{tabular}{cccccc} 
Objective function & Retrieval model & P@ 1 & P@5 & MAP & Macro AP@5 \\
\hline$C_{F}(\theta), C_{G}(\theta), C_{I}(\theta)$ (centroid) & Query image similarity & 3.00 & 2.40 & 1.15 & 13.35 \\
$C_{F}(\theta), C_{G}(\theta), C_{I}(\theta)$ (centroid) & Standard & 3.00 & 2.40 & 1.15 & 14.78 \\
$C_{F}(\theta), C_{G}(\theta), C_{I}(\theta)$ (centroid) & Image-to-text-to-image & 2.00 & 1.80 & 0.90 & 18.15 \\
\hline$C_{F}(\theta), C_{G}(\theta)$ & Proposed & 10.00 & 4.60 & 3.21 & 43.90 \\
$C_{F}(\theta), C_{G}(\theta), C_{I}(\theta)$ (medoid) & Proposed & 9.00 & 4.80 & 3.67 & 45.29 \\
$C_{F}(\theta), C_{G}(\theta), C_{I}(\theta)$ (centroid) & Proposed & $\mathbf{1 1 . 0 0}$ & $\mathbf{6 . 2 0}$ & $\mathbf{4 . 0 6}$ & $\mathbf{5 0 . 1 1}$ \\
\hline
\end{tabular}

$K=1,5$, where we not express relevance as a yes-or-no question (0 or 1 ), but rather as a percentage of present required attributes.

Finally, we provide a thorough analysis of the induced common, multimodal space and the inferred intermodal representations.

\section{RESULTS AND DISCUSSION}

\subsection{Quantitative Evaluation}

Our multimodal search results are presented in Table 1. These results show that our proposed multimodal retrieval model outperforms all baseline retrieval models. Our proposed retrieval model surpasses the image-to-text-to-image retrieval model by $450 \%$ on precision@1, by $244 \%$ on precision@5, by $351 \%$ on MAP, and by 176\% on macro AP@5. Compared to the standard retrieval model, our proposed retrieval model achieves an increase of $267 \%$ on precision@1, of $158 \%$ on precision@ 5 , of $253 \%$ on MAP, and of $239 \%$ on macro AP@5.

The standard multimodal retrieval model is very intuitive and actually creates the intermodal representation of the desired image based on the multimodal query. However, it lacks a mechanism to focus on retrieving the desired fashion attributes. Only calculating an overall cosine similarity between the desired image and the candidate images is not sufficient to return relevant images exhibiting both the query image attributes and the query text attributes. The image-to-text-to-image multimodal retrieval model on the other hand, by performing image search with a textual query composed of the main query image attributes and the query text attributes, does put focus on finding candidate images exhibiting the fashion attributes requested by the multimodal query. However, Laenen et al. [12] demonstrated that both for image annotation and image search the model still has difficulties with distinguishing between visually similar fashion attributes. Consequently, these two steps, i.e., image annotation and image search, both introduce errors into the search process. Our proposed multimodal retrieval model prefers to go from the query image to the candidate image directly, and computes their visual similarity as the aggregation of the cosine similarities of corresponding query and candidate image fragments. In contrast to the image-to-text-to-image retrieval model, our proposed retrieval model avoids the introduction of noise in the search process due to the additional image annotation step, at the cost of possibly not retrieving every desired query image attribute. To satisfy the query text attributes, our proposed multimodal retrieval model uses the cosine similarity of a query text fragment and candidate image fragment to explicitly require every query text attribute to be present in the retrieved images. This approach is different from the one in the
Table 2: Multimodal search results. Comparison of performance of our best model for different textual attributes. Training set frequency is the number of times the textual attribute occurs in the training set, multimodal query frequency is the number of multimodal queries containing the textual attribute and attributeP@K is precision@ $K$ for each individual textual attribute.

\begin{tabular}{|c|c|c|c|}
\hline Attribute & $\begin{array}{l}\text { Training set } \\
\text { frequency }\end{array}$ & $\begin{array}{l}\text { Multimodal query } \\
\text { frequency }\end{array}$ & $\begin{array}{l}\text { Attribute } \\
\text { P@5 }\end{array}$ \\
\hline wedding & 30566 & 1 & 100.00 \\
\hline $\operatorname{maxi}$ & 4579 & 2 & 100.00 \\
\hline V-neck & 6700 & 7 & 97.14 \\
\hline red & 2322 & 2 & 90.00 \\
\hline black & 5212 & 8 & 87.50 \\
\hline strapless & 11441 & 5 & 84.00 \\
\hline applique & 1446 & 1 & 80.00 \\
\hline mesh & 1739 & 1 & 80.00 \\
\hline lace & 21658 & 4 & 80.00 \\
\hline floral print & 682 & 2 & 80.00 \\
\hline sleeveless & 13306 & 11 & 78.18 \\
\hline short & 7658 & 9 & 77.78 \\
\hline short sleeves & 1158 & 10 & 74.00 \\
\hline wide & 2017 & 2 & 70.00 \\
\hline crystals & 2189 & 2 & 70.00 \\
\hline wear to work & 129 & 2 & 70.00 \\
\hline white & 4171 & 5 & 68.00 \\
\hline knee length & 3041 & 5 & 64.00 \\
\hline rhinestones & 1082 & 2 & 60.00 \\
\hline green & 754 & 2 & 60.00 \\
\hline scoop & 2369 & 2 & 60.00 \\
\hline evening & 20019 & 1 & 60.00 \\
\hline pink & 1248 & 1 & 60.00 \\
\hline summer & 2669 & 1 & 60.00 \\
\hline vertical stripes & 8 & 1 & 60.00 \\
\hline : & : & $\vdots$ & : \\
\hline winter & 1345 & 1 & 0.00 \\
\hline velvet & 446 & 1 & 0.00 \\
\hline khaki & 26 & 1 & 0.00 \\
\hline linen & 553 & 1 & 0.00 \\
\hline cut outs & 62 & 1 & 0.00 \\
\hline leopard print & 99 & 1 & 0.00 \\
\hline horizontal stripes & 10 & 2 & 0.00 \\
\hline cheetah print & 340 & 1 & 0.00 \\
\hline
\end{tabular}

image-to-text-to-image retrieval model, which does put focus on retrieving the requested fashion attributes, but does not explicitly impose that attributes in the generated textual query are exhibited in the returned images. When comparing the macro AP@5 of the different retrieval models, it is clear that the method employed in our proposed multimodal retrieval model for returning images satisfying the multimodal query texts is the most successful one. Finally, our proposed multimodal retrieval model has the benefit of having a regulator term to steer the model to give equal importance to the query image and query text when retrieving candidate images. In contrast, the standard and image-to-text-to-image retrieval models by definition assign more importance to the query images. The standard retrieval model builds the intermodal representation 
of the desired image from the representation of the query image, and the image-to-text-to-image retrieval model generates a textual description which contains much more text fragments related to the query image than related to the query text.

Moreover, the results in Table 1 indicate that the intermodal representations trained with the complete objective function, i.e., including $C_{I}(\theta)$, and using the cluster centroids to fulfill the role of the $\hat{c_{i}}$ in $C_{I}(\theta)$ provide the best multimodal search results. Hence, our best model is the proposed multimodal retrieval model operating on the aforementioned intermodal representations.

Overall, the multimodal search results for our best model remain quite low. The method employed to evaluate the search results is rather strict. It requires that all query image and query text attributes are displayed in the candidate image for it to be relevant. However, as dresses are characterized by a multitude of fashion attributes, it might very well be that for some multimodal queries there are no relevant images in our relatively small test set. In addition, the proposed multimodal retrieval model will retrieve images which overall are as similar as possible to the query image and which satisfy the query text. However, this might be inadequate to request all necessary query image attributes to be maintained in the retrieved images.

\subsection{Qualitative Evaluation}

We further study our results qualitatively to assess our model's performance. Figure 2 displays the images retrieved for 6 multimodal queries. Multimodal query "Find similar images but wear to work" requests dresses which are more suitable for the office than the query image, which is more casual. The first, second and fourth dress seem appropriate to wear to work, but this is rather subjective. The second dress also preserves the query image attributes (jewel neckline, sleeveless, short, black) and is considered as the only relevant image. The first dress has the wrong neckline (notched) and sleeve length (short sleeves), the fourth dress has the wrong sleeve length (short sleeves), dress length (knee length) and color (burgundy). For multimodal query "Find similar images but with a V-neck" the first dress is relevant (V-neck, black, white, sleeveless, sheath, short, casual). All other dresses have the requested query text attribute "V-neck", but do not have all query image attributes. For multimodal query "Find similar images but red" only the fifth dress is relevant (red, V-neck, sleeveless, long, rhinestones, evening). All other dresses are red too, but do not have all required query image attributes. Multimodal query "Find similar images but shift" retrieves two dresses with this shape: the first and the fifth. None of the dresses have all requested attributes (shift, jewel neckline, short sleeves, short, pink, floral print, summer). However, each dress has some of the required attributes and can be considered similar to the query image. For multimodal query "Find similar images but with rhinestones" all dresses except the fifth one have rhinestones. None of the retrieved dresses is relevant though, as none of them have the right color (white). However, except for the color, the first dress has all requested attributes (rhinestones, strapless, sweetheart, long, A-line, pleated, bridesmaid). Multimodal query "Find similar images but strapless and short" returns three relevant dresses: the first, second and third. They are all red, strapless, short, accented with an ornament at their waist and suitable for a bridesmaid, as requested by the multimodal query. For these multimodal query results we can compute refined precision@1 and refined precision@5 scores, expressing relevance as a percentage of present required attributes. From left to right and top to bottom, refined precision@1 is respectively $60 \%, 100 \%, 66.67 \%, 71.43 \%, 87.50 \%$, and $100 \%$, and refined precision@5 is respectively 48\%,71.43\%, 76.67\%, 57.14\%, 77.14\% and $80 \%$. We conclude that, although our proposed multimodal retrieval model does not have the ability to explicitly focus on retrieving all query image attributes, it is nevertheless capable of retrieving relevant candidate images for the provided multimodal queries.

\subsection{Discussion of the Proposed Alignment and Retrieval Models}

Our multimodal search results indicate that the neural network has learned what certain fashion attributes look like. Hence, we can analyze the 1000-dimensional intermodal representations to acquire insight in the meaning of their different components.

When we project the image fragments of the test set images and the Zappos phrases to the common, multimodal space, we clearly see two clusters: one containing all image fragments and one containing all Zappos phrases. Hence, even in the common, multimodal space the two modalities remain separated.

Within the cluster of image fragments, we observe that image fragments showing the same part of a dress are close-by, e.g., the tops are grouped together. Additionally, similar parts are closer together than dissimilar parts, e.g., necklines and tops are closer together than tops and skirts.

When looking at the intermodal representations of the Zappos phrases, both visually and semantically similar textual attributes appear to be close to each other. Textual attributes which are semantically similar but differ in appearance are further apart. For example, the colors "coral", "pink" and "orange" are very close to each other, but are further away from other colors like "brown" and "gray". Also, necklines "scoop", "square neck" and "off the shoulder" are close to each other, but further away from "sweetheart", which is very close to "strapless". In contrast, in the original word embedding space semantically similar textual attributes are close to each other, regardless of appearance.

In the intermodal representations of textual attributes a component is either zero or positive (a consequence of using the ReLU activation function). Actually, for the textual attributes in our vocabulary, we observe that only a few of the 1000 components are non-zero. This indicates that our model has learned which components of the image fragments to focus on when looking for a specific fashion attribute. For example, neckline "sweetheart" only has 7 non-zero components. Hence, image fragments in the common, multimodal space with positive values for these components will result in a positive inner product with "sweetheart" and thus are expected to show a sweetheart neckline. An interesting observation can be made regarding the number of non-zero components of fashion attribute "dress". The intermodal representation of "dress" is actually an all-zero vector. This happens because every image in the Amazon Dresses dataset is described with the text fragment "dress". Therefore, the neural network was unable to learn which components are indicators for an image fragment showing a dress. It solves this nicely by assigning the all-zero vector to "dress", which 



Find similar images but with rhinestones
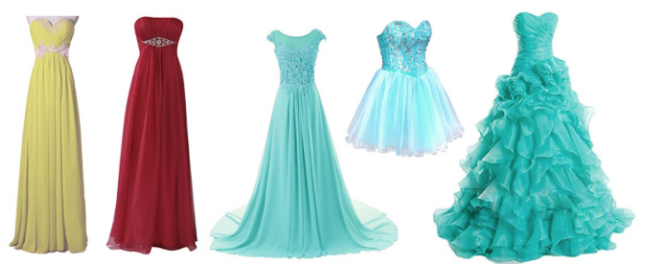
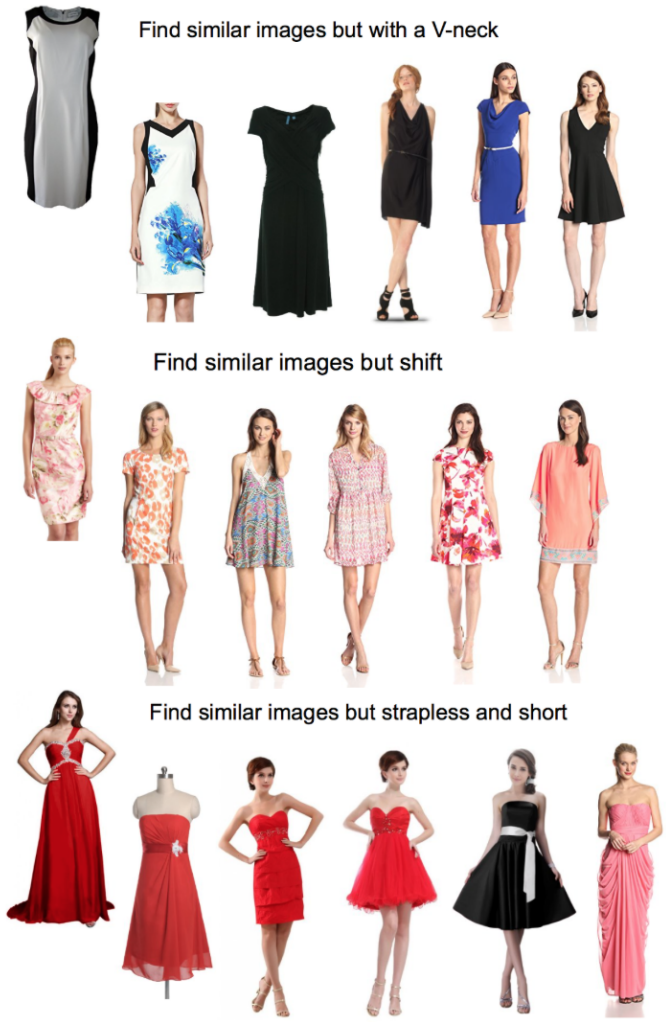

Figure 2: Multimodal search examples ${ }^{1}$. Six examples of multimodal queries for which our model performs well. For each multimodal query, the top 5 retrieved images are shown.
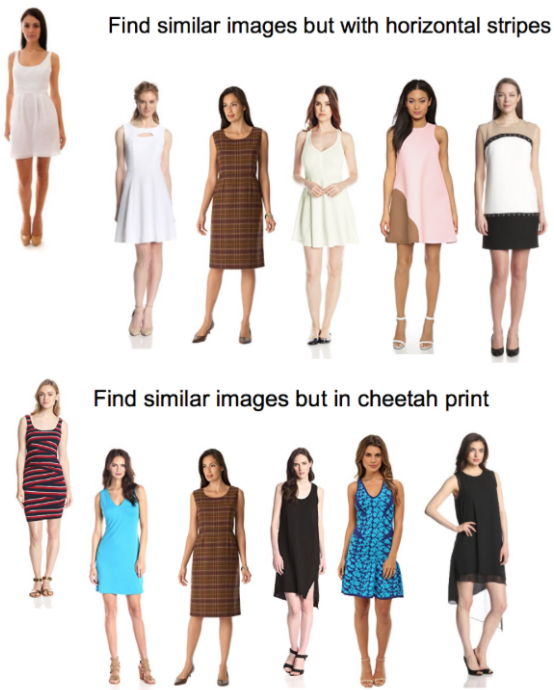

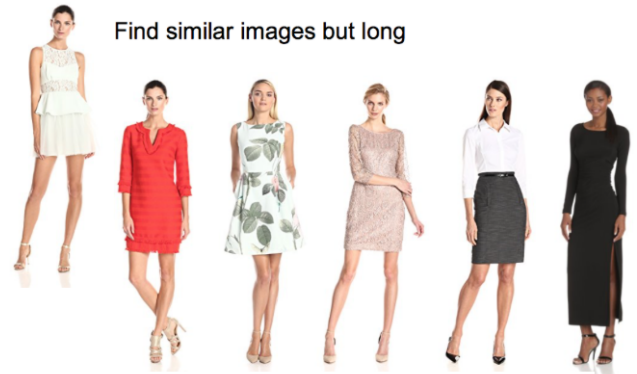

Find similar images but sleeveless and in linen


Figure 3: Multimodal search examples ${ }^{1}$. Four examples of multimodal queries for which our model returns bad results. For each multimodal query, the top 5 retrieved images are shown. 
produces an inner product of 0 with each image fragment because everything is a dress. Additionally, we observe that there are 237 components in the intermodal representations which are zero for all textual attributes. Corresponding components in the image fragments never matter when looking for the presence of certain fashion attributes. Hence, these components represent characteristics of the image fragments which are not indicators for any fashion attributes in our current vocabulary.

Visually similar fashion attributes share some of their non-zero components. This is natural, as some of the same components matter when determining the presence of these fashion attributes. For example, "strapless" has 10 non-zero components of which 3 are also non-zero for "sweetheart". This explains why the neural network model of Laenen et al. [12] sometimes has trouble distinguishing between visually similar fashion attributes: a large value for a shared component might produce a positive inner product for an incorrect fashion attribute. Multimodal query "Find similar images but long" in Figure 3 suffers from this problem. Instead of retrieving long dresses, it retrieves dresses with longer sleeves. The intermodal representation of "long" only has 4 non-zero components, of which one is shared with "long sleeves". This shared component is probably high in the intermodal representation of the retrieved images because of their sleeve length, which produces the incorrect positive inner product regarding the dress length. Note that the opposite, i.e., mistaking "long sleeves" for "long" is less probable, as "long sleeves" has 40 non-zero components.

In other cases, our proposed multimodal retrieval model makes errors because the intermodal representations of certain fashion attributes are underdeveloped. For example, Figure 3 shows bad results for multimodal queries asking for a different print ("horizontal stripes", "cheetah print") and another fabric ("linen"). Fashion attribute "horizontal stripes" only occurs 10 times in the training set, which is not enough for the neural network to learn what this print looks like. The results for the multimodal query asking for "horizontal stripes" illustrate that the model is unable to retrieve images with a horizontally striped print. Also for other fashion attributes which occur infrequently in the training set, e.g., "khaki" and "cut outs", we observe that our retrieval model cannot find candidate images exhibiting these attributes (Table 2). Print "cheetah print" suffers from noise in the dataset. It occurs 340 times in the training set, but most of the time it is listed as another possibility for the print of the displayed dresses. This noise in the product descriptions is very obstructive and prevents the neural network from learning what a cheetah print looks like. Finally, we observe that the intermodal representations for fabrics, e.g., linen, are also of low quality. The reason for this is that in general fabrics are very difficult to identify from an image, even for humans.

\section{CONCLUSION}

In this paper, we have proposed a novel search paradigm to search fashion e-commerce data called multimodal search. Our multimodal queries consist of an e-commerce image of a clothing item together with text that modifies the image or adds attributes to it. We proposed a multimodal retrieval model which operates on intermodal representations of fashion attributes obtained with a neural network, and which exploits the semantic similarity information contained in their inner product. We demonstrated that the proposed multimodal retrieval model outperforms state-of-the-art models, but the retrieval of the query image attributes can still be improved. This will be the focus of our future research. Additionally, we will expand our work to allow street images as query images, i.e., images taken with a smartphone or found on social media. Moreover, it would be interesting to add additional context to this work such as co-purchase or co-click data in order to improve our image and text features $[2,5]$.

\section{REFERENCES}

[1] S. Bell and K. Bala. 2015. Learning Visual Similarity for Product Design with Convolutional Neural Networks. ACM Transactions on Graphics (TOG) 34, 4 (July 2015).

[2] N. Djuric, H. Wu, V. Radosavljevic, M. Grbovic, and N. Bhamidipati. 2015. Hierarchical Neural Language Models for Joint Representation of Streaming Documents and Their Content. In Proceedings of the 24th International Conference on World Wide Web $(W W W)$. 248-255.

[3] J. Donahue, L. A. Hendricks, S. Guadarrama, M. Rohrbach, S. Venugopalan, K. Saenko, and T. Darrell. 2015. Long-Term Recurrent Convolutional Networks for Visual Recognition and Description. In The IEEE Conference on Computer Vision and Pattern Recognition (CVPR).

[4] A. Ghodrati, X. Jia, M. Pedersoli, and T. Tuytelaars. 2015. Swap Retrieval: Retrieving Images of Cats When the Query Shows a Dog. In Proceedings of the 5th ACM International Conference on Multimedia Retrieval (ICMR). 395-402.

[5] M. Grbovic, V. Radosavljevic, N. Djuric, N. Bhamidipati, J. Savla, V. Bhagwan, and D. Sharp. 2015. E-commerce in Your Inbox: Product Recommendations at Scale. In Proceedings of the 21th ACM SIGKDD International Conference on Knowledge Discovery and Data Mining. 1809-1818.

[6] X. Han, Z. Wu, P. X. Huang, X. Zhang, M. Zhu, Y. Li, Y. Zhao, and L. S. Davis. 2017. Automatic Spatially-Aware Fashion Concept Discovery. In The IEEE International Conference on Computer Vision (ICCV).

[7] J.-H. Hsiao and L.-J. Li. 2014. On Visual Similarity based Interactive Product Recommendation for Online Shopping. In The IEEE International Conference on Image Processing (ICIP).

[8] Y. Jia, E. Shelhamer, J. Donahue, S. Karayev, J. Long, R. Girshick, S. Guadarrama, and T. Darrell. 2014. Caffe: Convolutional Architecture for Fast Feature Embedding. In Proceedings of the 22nd ACM International Conference on Multimedia (ACMMM). 675-678.

[9] Y. Kalantidis, L. Kennedy, and L.-J. Li. 2013. Getting the Look: Clothing Recognition and Segmentation for Automatic Product Suggestions in Everyday Photos. In Proceedings of International Conference on Multimedia Retrieval (ICMR).

[10] A. Karpathy and L. Fei-Fei. 2015. Deep Visual-Semantic Alignments for Generating Image Descriptions. In The IEEE Conference on Computer Vision and Pattern Recognition (CVPR). 3128-3137.

[11] M. H. Kiapour, X. Han, S. Lazebnik, A. C. Berg, and T. L. Berg. 2015. Where to Buy It: Matching Street Clothing Photos in Online Shops. In The IEEE International Conference on Computer Vision (ICCV). 3343-3351.

[12] K. Laenen, S. Zoghbi, and M-F. Moens. 2017. Cross-modal Search for Fashion Attributes. In Proceedings of the KDD 2017 Workshop on Machine Learning Meets Fashion.

[13] T. Mikolov, K. Chen, G. S. Corrado, and J. Dean. 2013. Efficient Estimation of Word Representations in Vector Space. CoRR abs/1301.3781 (2013).

[14] O. Vinyals, A. Toshev, S. Bengio, and D. Erhan. 2015. Show and Tell: A Neural Image Caption Generator. In The IEEE Conference on Computer Vision and Pattern Recognition (CVPR).

[15] K. Xu, J. Ba, R. Kiros, K. Cho, A. Courville, R. Salakhudinov, R. Zemel, and Y. Bengio. 2015. Show, Attend and Tell: Neural Image Caption Generation with Visual Attention. In Proceedings of the 32nd International Conference on Machine Learning (ICML). 2048-2057.

[16] F. Yang, A. Kale, Y. Bubnov, L. Stein, Q. Wang, H. Kiapour, and R. Piramuthu. 2017. Visual Search at eBay. In Proceedings of the 23rd ACM SIGKDD Conference on Knowledge Discovery and Data Mining. 2101-2110.

[17] B. Zhao, J. Feng, X. Wu, and S. Yan. 2017. Memory-Augmented Attribute Manipulation Networks for Interactive Fashion Search. In IEEE Conference on Computer Vision and Pattern Recognition (CVPR). 6156-6164.

[18] S. Zoghbi, G. Heyman, J. C. Gomez, and M-F. Moens. 2016. Fashion Meets Computer Vision and NLP at e-Commerce Search. International fournal of Computer and Electrical Engineering (IFCEE) 8, 1 (February 2016), 31-43. 